\title{
Clinical, Radiologic, and Endoscopic Manifestations of Small Bowel Malignancies: a First Report from Thailand
}

\author{
Natthakan Tangkittikasem ${ }^{1}$, Thiraphon Boonyaarunnate ${ }^{2}$, Pitulak Aswakul ${ }^{3}$, \\ Udom Kachintorn ${ }^{1}$, Varayu Prachayakul ${ }^{1 *}$
}

\begin{abstract}
Background: The symptoms of small bowel malignancies are mild and frequently nonspecific, thus patients are often not diagnosed until the disease is at an advanced stage. Moreover, the lack of sufficient studies and available data on small bowel cancer makes diagnosis difficult, further delaying proper treatment for these patients. In fact, only a small number of published studies exist, and there are no studies specific to Thailand. Radiologic and endoscopic studies and findings may allow physicians to better understand the disease, leading to earlier diagnosis and improved patient outcomes. Objective: To retrospectively analyze the clinical, radiologic, and endoscopic characteristics of small bowel cancer patients in Thailand's Siriraj Hospital. Materials and Methods: This retrospective analysis included 185 adult patients $(97$ men, 88 women; mean age $=57.6 \pm 14.9$ ) with pathologically confirmed small bowel cancer diagnosed between January 2006 and December 2013. Clinical, radiologic, and endoscopic findings were collected and compared between each subtype of small bowel cancer. Results: Of the 185 patients analyzed, gastrointestinal stromal tumor (GIST) was the most common diagnosis $(39.5 \%, \mathrm{n}=73)$. Adenocarcinoma was the second most common $(25.9 \%, \mathrm{n}=48)$, while lymphoma and all other types were identified in $24.3 \%(n=45)$ and $10.3 \%(n=19)$ of cases, respectively. The most common symptoms were weight loss (43.2\%), abdominal pain (38.4\%), and upper gastrointestinal bleeding (23.8\%). Conclusions: Based on radiology and endoscopy, this study revealed upper gastrointestinal bleeding, an intra-abdominal mass, and a sub-epithelial mass as common symptoms of GIST. Obstruction and ulcerating/circumferential masses were findicative of adenocarcinoma, as revealed by radiology and endoscopy, respectively. Finally, no specific symptoms were related to lymphoma.
\end{abstract}

Keywords: Small bowel cancer - GIST - adenocarcinoma - lymphoma - metastasis - radiologic manifestation

Asian Pac J Cancer Prev, 16 (18), 8613-8618

\section{Introduction}

Small bowel cancer is a rare malignancy that accounts for only $2 \%$ of all gastrointestinal cancers (Paski, 2009; Pan et al, 2011). The incidence of malignant small intestine tumors, which ranges from $0.5-1.5 / 100,000$ in men and $0.2-1.0 / 100,000$ in women (Parkin et al., 1992), seems to be higher in North America and Europe than Asia (Haselkorn et al., 2005). Most cases of small bowel cancer are diagnosed late in the course of the disease because of its vague clinical presentations (e.g. abdominal pain and abdominal discomfort), and patients who present with obvious clinical manifestations such as gastrointestinal bleeding, intestinal obstruction, or ascites are almost always diagnosed with advanced stage cancer. The most common malignancy lesions are adenocarcinoma, neuroendocrine tumor (NET), sarcomas, and small bowel lymphoma. However, the incidence of small bowel cancer is increasing, particularly for carcinoid tumor (Islam, 2014). The small bowel is not a common location of metastatic cancers, although there are many case reports and series discussing small bowel tumor metastasis from lung cancer (Liu et al., 2015), cervical cancer (Qiu et al., 2015), melanoma (Conversano et al., 2014; Kouladouros et al., 2015), renal cell carcinoma (Ismail et al., 2015; Gorski et al., 2015), and unknown adenocarcinoma (Alkabie et al., 2015). On average, a small bowel cancer diagnosis is made 18 months following presentation with the first symptom. Further complicating matters is the fact that the misdiagnosis rate is as high as 56\% (Zhou et al., 1999). The overall 1- and 5-year relative survival rates (RS) are $67.9 \%$ and $47.9 \%$, respectively (Anderson et al., 2015). Notably, the relative 5-year survival rate has increased over the past 5 years (from $40 \%$ to $47.9 \%$ ). The lowest relative survival rate occurred with adenocarcinoma, while lymphomas, sarcomas, and carcinoid tumors showed slightly better outcomes (Pan et al., 2011).

Small bowel cancer is classified into 40 different histological subtypes (Pan et al., 2011). Between 1985 and 2005, 67,843 cases of small bowel cancer were diagnosed in the United States, with the most prevalent subtypes being carcinoid tumor (37.4\%), adenocarcinoma 
Natthakan Tangkittikasem et al

(36.9\%), lymphoma (17.3\%), and sarcoma (8.4\%) (Haselkorn et al., 2005). Similarly, Zhi-Wei Zhou and colleagues (1999) reported that, of the 75 patients who were diagnosed with small bowel cancer in China between 1964 and 1995, 33.33\% had adenocarcinoma, 32.91\% had leiomyosarcoma, and $26.67 \%$ had lymphoma. Not only is small bowel cancer rarer in China than in the United States, the most prevalent subtype was not even diagnosed in China.

Predisposing factors for small bowel cancer are Crohn disease, with a relative risk (RR) of 33.0 (95\% CI, 15-60.9) (Canavan et al., 2006). A recent meta-analysis demonstrated that the RR for individuals consuming the highest versus lowest category of alcohol intake was 1.51 (95\% CI, 0.83-2.75). The pooled RR for individuals in the highest versus lowest category of smoking was 1.24 (95\% CI, 0.71-2.17). In relation to dietary factors, high fiber intake and normal body weight may be protective, while high intake of red/processed meat and sugary drinks may increase risk (Bennett et al., 2015).

Diagnosing small bowel cancer is difficult given the investigation relies on the location of the tumors. Upper gastrointestinal study with small bowel followthrough, ultrasonography of the whole abdomen, computed tomography (CT) scan, CT and enteroclysis/ enterography, video capsule enteroscopy (VCE), esophagogastroduodenoscopy (EGD), and balloonassisted enteroscopy (BAE) were commonly used to determine localization of the tumor and to evaluate adjacent organs (Islam et al., 2014).

To the best of our knowledge, there are no published studies detailing small bowel cancer rates, especially in Southeast Asia, including Thailand. A better understanding of clinical, radiologic, and endoscopic markers for small bowel cancer may allow earlier diagnoses and improved patient outcomes. Therefore, we sought to study the clinical, radiologic, and endoscopic characteristics of small bowel cancer patients in patients who sought treatment at Thailand's Siriraj Hospital.

\section{Materials and Methods}

This retrospective review was approved by the Siriraj Internal Review Board (SIRB) for ethical issues. Informed consent was obtained from all patients who underwent the procedures. The data for this study were obtained from Siriraj Hospital's electronic medical record program. Adult patients were diagnosed with small bowel cancer based on pathological examination between January 2006 and December 2013 at Siriraj Hospital. Baseline characteristics, clinical findings, radiologic findings, and endoscopic findings were collected and compared between each subtype of small bowel cancer.

Tumor characteristics were analyzed using descriptive statistics, including frequencies and percentages for categorical variables. Continuous variables were reported as means, standard deviation of normally distributed variables, and median, minimum, and maximum of abnormally distributed variables. The normality of distribution of the variables was examined using the Kolmogorov-Smirnov test. The association of normally and abnormally distributed continuous variables was compared between three groups using one-way ANOVA and the Kruskal-Wallis $H$ test, respectively. The association of categorical variables was compared between four groups using the chi-square test or Fisher's exact test.

\section{Results}

The 185 small bowel cancer patients enrolled in the study were classified into four major histological subtypes: gastrointestinal stromal tumor (GIST), adenocarcinoma, lymphoma, and other. GIST was the most common subtype, accounting for $39.5 \%$ of cases $(n=73)$. Adenocarcinoma, lymphoma, and other were $25.9 \%(n=48), 24.3 \%(n=45)$, and $10.3 \%(\mathrm{n}=19)$, respectively

(Table 1). The mean age at diagnosis for overall tumor was 57.6 years, with the diagnosis being more common in men. Some patients had associated cancers (e.g. colon

Table 1. Baseline Characteristics of Patients With Small Bowel Cancer.

\begin{tabular}{|c|c|c|c|c|c|}
\hline Characteristic & $\begin{array}{c}\text { GIST } \\
(n=73)\end{array}$ & $\begin{array}{l}\text { Adenocarcinoma } \\
\qquad(\mathrm{n}=48)\end{array}$ & $\begin{array}{l}\text { Lymphoma } \\
\qquad(\mathrm{n}=45)\end{array}$ & $\begin{array}{l}\text { Other } \\
(n=19)\end{array}$ & $\mathrm{P}$ \\
\hline Age $(y)$, mean + SD & $54.4+13.9$ & $63.2+13.9$ & $55.6+16.5$ & $60.4+14$ & 0.008 \\
\hline Female, n $(\%)$ & $41(56.2)$ & $22(45.8)$ & $17(37.8)$ & $8(42.1)$ & 0.243 \\
\hline Smoking, n (\%) & $11(15.1)$ & $14(29.2)$ & $6(13.3)$ & $7(36.8)$ & 0.229 \\
\hline Alcoholic drinking, n (\%) & $14(19.2)$ & $13(27.1)$ & $9(20)$ & $9(47.4)$ & 0.238 \\
\hline Hct $(\%)$, mean + SD & $30.8+8.1$ & $30.0+6.1$ & $31.6+7.6$ & $31.1+9.3$ & 0.795 \\
\hline \multicolumn{6}{|l|}{ Associated disease } \\
\hline Breast cancer & 1 & 1 & - & - & \\
\hline Colon cancer & - & 2 & - & $3 *$ & \\
\hline Ovary cancer & - & 1 & - & - & \\
\hline Renal cell carcinoma & - & - & - & $2^{\#}$ & \\
\hline Lung cancer & - & 1 & - & $1^{\$}$ & \\
\hline Nasopharynx cancer & 1 & - & - & - & \\
\hline AML & 1 & - & - & - & \\
\hline Malignant hemangiopericytoma & 1 & - & - & - & \\
\hline HIV infection & - & - & 3 & - & \\
\hline Unknown cancer & - & - & - & $1^{\&}$ & \\
\hline
\end{tabular}

*Colon cancer was found in two patients with neuroendocrine tumor and one metastasis; ${ }^{\#}$ Two patients with renal cell carcinoma had small bowel metastasis; ${ }^{\$}$ One patient with lung cancer had small bowel metastasis; ${ }^{\star}$ One patient with unknown primary cancer with had small bowel metastasis. GIST, gastrointestinal stromal tumor 
Clinical, Radiologic, and Endoscopic Manifestations of Small Bowel Malignancies: a First Report from Thailand

cancer, breast cancer, ovarian cancer, and lung cancer). The overall baseline hematocrit level was $30.8 \%$. Neither smoking nor alcohol intake increased the risk of small bowel cancer (Table 1). Metastasis to the small bowel was observed in three patients (lung cancer [1 case] and renal cell carcinoma [2 cases]), while the prevalence of small bowel cancer increased between 2006 and 2012, as shown in Figure 1. In the present study, small bowel cancer was most common between the ages of 61 and 70 years (Figure 2).

\section{Clinical manifestations}

Of all cases, only $4.9 \%$ of the patients presented as asymptomatic. A total of $29 \%$ of patients revealed unremarkable findings on physical examination. The most common clinical manifestations related to GIST

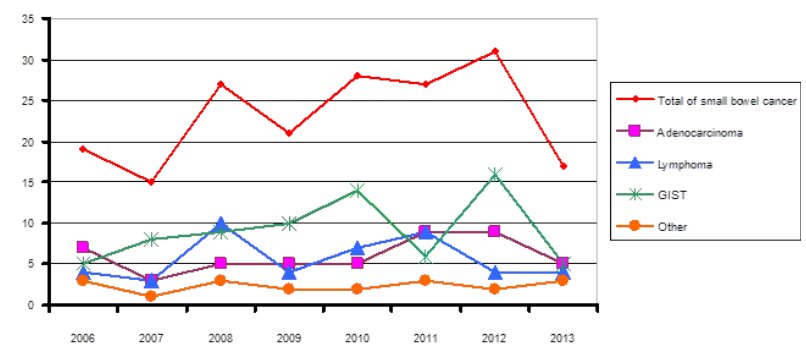

Figure 1. Prevalence of Small Bowel Cancer. GIST; Gastrointestinal stromal tumor

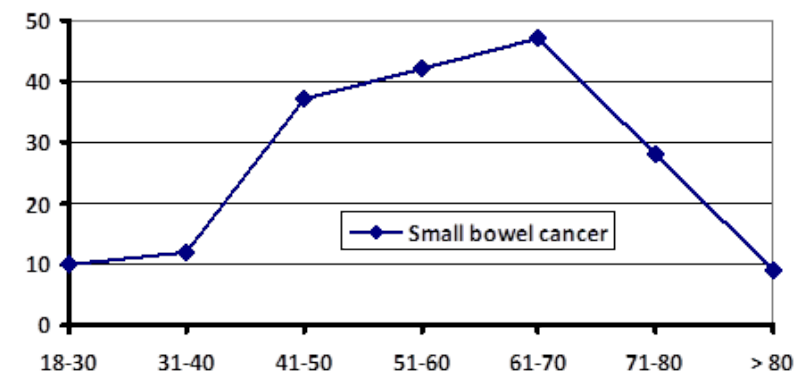

Figure 2. Average Age of Participants.

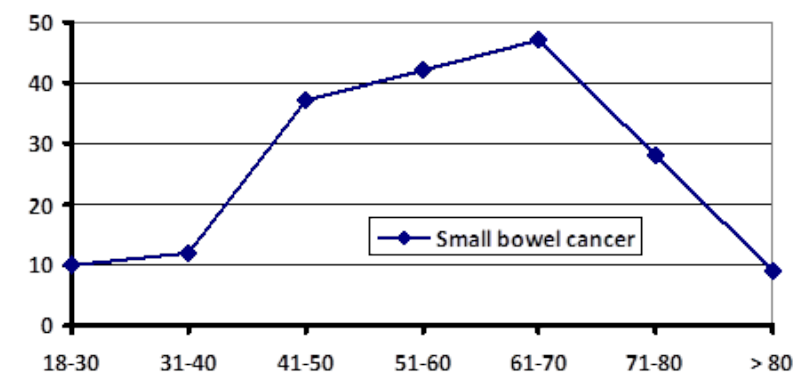

Figure 3. Types of Tumor Mass Evaluated by Ultrasound were anemia and upper gastrointestinal bleeding $(24.7 \%$ and $42.5 \%$, respectively), while weight loss and intestinal obstruction were predominant in small bowel lymphoma and adenocarcinoma for 57.8\%/24.4\% and $77.1 \% / 29.2 \%$, respectively (Table 2).

\section{Radiologic findings}

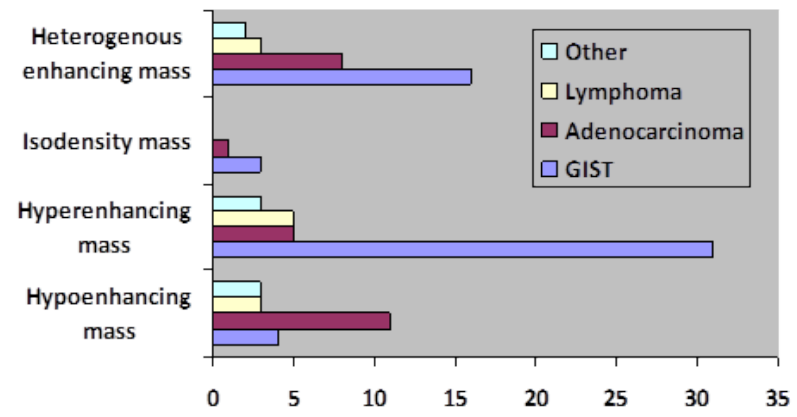

Figure 4. Types of Tumor Mass Evaluated by Computed Tomography. GIST; Gastrointestinal stromal tumor

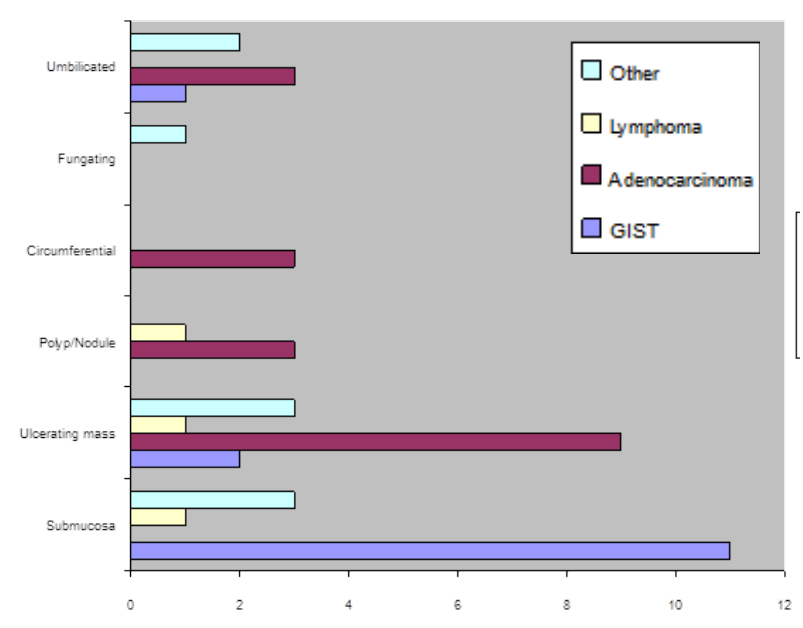

Figure 5. Types of Tumor Mass Evaluated by Esophagogastroduodenoscopy. GIST; Gastrointestinal stromal tumor

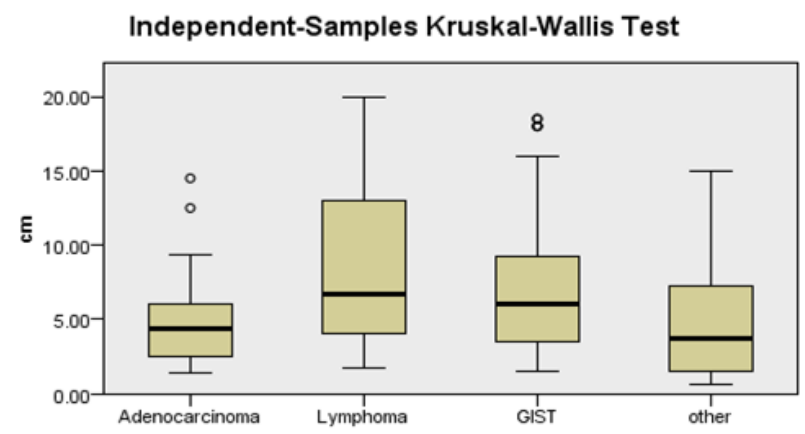

Figure 6. Average Tumor Size by Tumor Type. GIST; Gastrointestinal stromal tumor

Table 2. Detection of Abdominal Masses by Ultrasound

\begin{tabular}{|c|c|c|c|c|c|c|c|c|c|}
\hline & \multicolumn{3}{|c|}{ GIST $(n=16)$} & \multicolumn{3}{|c|}{ Adenocarcinoma $(\mathrm{n}=9)$} & \multicolumn{3}{|c|}{ Lymphoma $(\mathrm{n}=14)$} \\
\hline & No. & Sensitivity (\%) & Specificity (\%) & No. & Sensitivity (\%) & Specificity (\%) & No. & Sensitivity (\%) & Specificity $(\%)$ \\
\hline Mass & 15 & 93.8 & 48.2 & 4 & 44.4 & 26.5 & 8 & 57.1 & 27.6 \\
\hline
\end{tabular}

GIST, gastrointestinal stromal tumor 
Table 3. Clinical Manifestations in Patients with Small Bowel Cancer

\begin{tabular}{|c|c|c|c|c|c|}
\hline Clinical manifestation & $\begin{array}{c}\text { Total } \\
(\mathrm{n}=185)\end{array}$ & $\begin{array}{c}\text { GIST } \\
(n=73)\end{array}$ & $\begin{array}{l}\text { Adenocarcinoma } \\
\qquad(\mathrm{n}=48)\end{array}$ & $\begin{array}{l}\text { Lymphoma } \\
\qquad(\mathrm{n}=45)\end{array}$ & $\mathrm{P}$ \\
\hline \multicolumn{6}{|l|}{ Onset } \\
\hline Median (mo) & & 6 & 2 & 3 & \\
\hline Minimum (d) & & 1 & 3 & 1 & \\
\hline Maximum (mo) & & 120 & 14 & 36 & \\
\hline Asymptomatic, n (\%) & $9(4.9)$ & $8(11)$ & $0(0)$ & $1(2.2)$ & 0.014 \\
\hline Anemia, n $(\%)$ & $29(15.7)$ & $18(24.7)$ & $6(12.5)$ & $5(11.1)$ & 0.095 \\
\hline Abdominal mass, $\mathrm{n}(\%)$ & $15(8.1)$ & $8(11.1)$ & $0(0)$ & $7(15.6)$ & 0.008 \\
\hline Weight loss, n (\%) & $80(43.2)$ & $17(23.3)$ & $37(77.1)$ & $26(57.8)$ & $<0.001$ \\
\hline \multicolumn{6}{|l|}{ Amount of weight loss $(\mathrm{kg} / \mathrm{mo})$} \\
\hline Median & & 1.5 & 4.8 & 3 & \\
\hline Minimum & & 0.3 & 0.5 & 0.33 & \\
\hline Maximum & & 13 & 18 & 20 & \\
\hline Abdominal pain, n (\%) & $71(38.40)$ & $25(34.2)$ & $19(39.6)$ & $27(60)$ & 0.02 \\
\hline Abdominal discomfort, $\mathrm{n}(\%)$ & $39(21.0)$ & $7(9.6)$ & $15(31.2)$ & $17(37.8)$ & 0.001 \\
\hline Lower GI bleeding, n (\%) & $8(4.3)$ & $4(5.5)$ & $0(0)$ & $4(8.9)$ & 0.093 \\
\hline Upper GI bleeding, n (\%) & $44(23.8)$ & $31(42.5)$ & $10(20.8)$ & $3(6.7)$ & $<0.001$ \\
\hline Intestinal obstruction, n (\%) & $26(14.0)$ & $1(1.4)$ & $14(29.2)$ & $11(24.4)$ & $<0.001$ \\
\hline Obstructive jaundice, n (\%) & $11(5.9)$ & $1(1.4)$ & $10(20.8)$ & $0(0)$ & $<0.001$ \\
\hline Peritonitis, $\mathrm{n}(\%)$ & $18(9.7)$ & $7(9.6)$ & $2(4.2)$ & $9(20)$ & 0.044 \\
\hline Normal examination, $\mathrm{n}(\%)$ & $54(29.1)$ & $35(47.9)$ & $11(22.9)$ & $8(17.8)$ & 0.001 \\
\hline
\end{tabular}

*GIST, gastrointestinal stromal tumor

Table 4. Computed Tomography Findings

\begin{tabular}{|c|c|c|c|c|c|c|c|c|c|}
\hline \multirow[t]{2}{*}{ Finding } & \multicolumn{3}{|c|}{$\operatorname{GIST}(\mathrm{n}=55)$} & \multicolumn{3}{|c|}{ Adenocarcinoma $(n=43)$} & \multicolumn{3}{|c|}{ Lymphoma $(\mathrm{n}=30)$} \\
\hline & No. & $\begin{array}{c}\text { Sensitivity } \\
(\%)\end{array}$ & $\begin{array}{c}\text { Specificity } \\
(\%)\end{array}$ & No. & $\begin{array}{c}\text { Sensitivity } \\
(\%)\end{array}$ & $\begin{array}{c}\text { Specificity } \\
(\%)\end{array}$ & No. & $\begin{array}{c}\text { Sensitivity } \\
(\%)\end{array}$ & $\begin{array}{c}\text { Specificity } \\
(\%)\end{array}$ \\
\hline Mass & 55 & 100 & 38.6 & 27 & 62.8 & 18 & 17 & 56.7 & 18.6 \\
\hline Bowel wall thickening & 3 & 5.5 & 68.2 & 12 & 27.9 & 81 & 14 & 46.7 & 85 \\
\hline Intestinal obstruction & 3 & 5.5 & 68.2 & 18 & 41.9 & 87 & 6 & 20 & 77.9 \\
\hline Intussusception & 0 & 0 & 94.3 & 1 & 2.3 & 96 & 4 & 13.3 & 99.12 \\
\hline Biliary obstruction & 2 & 3.6 & 84.1 & 11 & 25.6 & 95 & 2 & 6.7 & 87.6 \\
\hline Lymphadenopathy & 8 & 14.5 & 53.4 & 15 & 34.9 & 66 & 22 & 73.3 & 76.1 \\
\hline
\end{tabular}

GIST, gastrointestinal stromal tumor

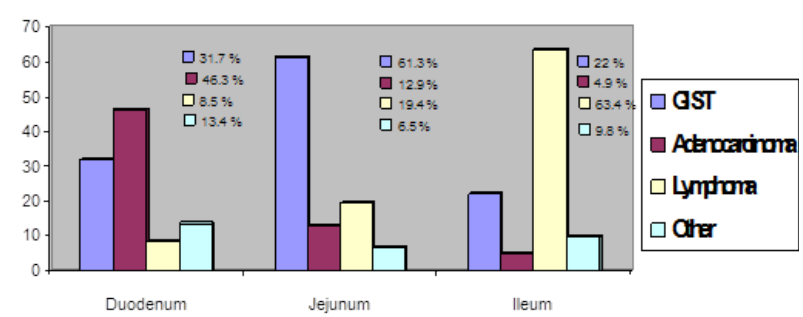

Figure 7. Tumor Location of Small Bowel Cancer by Subtype. GIST; Gastrointestinal stromal tumor

Ultrasound examination indicated that 29 patients had an abdominal mass (Figurere 3), including 15 patients who were diagnosed with GIST, 4 who were diagnosed with adenocarcinoma, and 8 who were diagnosed with lymphoma. Overall sensitivity and specificity for diagnosis of small bowel tumor by ultrasound was not acceptable save for GIST (detection of intra-abdominal mass) (Table 3). CT scans were performed for a total of 143 patients; findings were categorized as mass, bowel wall thickening, intestinal obstruction, intussusception biliary obstruction, or lymphadenopathy (Figure 4; Table 4).

\section{Detection by esophagogastroduodenoscopy}

Esophagogastroduodenoscopy (EGD) was performed on a total of 103 patients, with findings classified as intraluminal mass (Figure 5), ulcer, or gastrointestinal obstruction (Table 5).

\section{Tumor size}

Tumor size was measured for each tumor type (Figure 6). The median tumor size for adenocarcinoma, lymphoma, and GIST was $4.4 \mathrm{~cm}, 6.75 \mathrm{~cm}$, and $6.0 \mathrm{~cm}$, respectively. The maximum size was $14.5 \mathrm{~cm}, 20 \mathrm{~cm}$, and $18.5 \mathrm{~cm}$, respectively, and the minimum size was $1.4 \mathrm{~cm}$, $1.7 \mathrm{~cm}$, and $1.5 \mathrm{~cm}$, respectively.

\section{Site of small bowel cancer}

For each histological subtype, the most common histological subtype was recorded. The most common site for GIST was the jejunum, for adenocarcinoma it was the duodenum, and for lymphoma it was the ileum.

\section{Discussion}


Table 5. Detection of Small Bowel Cancer by Esophagogastroduodenoscopy

\begin{tabular}{|c|c|c|c|c|c|c|c|c|c|}
\hline \multirow[t]{2}{*}{ Finding } & \multicolumn{3}{|c|}{$\operatorname{GIST}(n=41)$} & \multicolumn{3}{|c|}{ Adenocarcinoma $(\mathrm{n}=31)$} & \multicolumn{3}{|c|}{ Lymphoma $(\mathrm{n}=20)$} \\
\hline & No. & $\begin{array}{c}\text { Sensitivity } \\
(\%)\end{array}$ & $\begin{array}{c}\text { Specificity } \\
(\%)\end{array}$ & No. & $\begin{array}{c}\text { Sensitivity } \\
(\%)\end{array}$ & $\begin{array}{c}\text { Specificity } \\
(\%)\end{array}$ & No. & $\begin{array}{c}\text { Sensitivity } \\
(\%)\end{array}$ & $\begin{array}{c}\text { Specificity } \\
(\%)\end{array}$ \\
\hline Mass & 14 & 34.1 & 45.2 & 21 & 67.7 & 62.5 & 4 & 20 & 47 \\
\hline Ulcer & 3 & 7.3 & 91.9 & 4 & 12.9 & 94.4 & 1 & 5 & 91.7 \\
\hline Obstruction & 0 & 0 & 74.2 & 15 & 48.4 & 98.6 & 1 & 5 & 81.9 \\
\hline
\end{tabular}

*GIST, gastrointestinal stromal tumor

Small bowel cancer is very rare. From 2006 to 2013, 185 patients were diagnosed with small bowel cancer at Siriraj Hospital. Within this timeframe, there was a notable increase in diagnoses between 2006 and 2012, with a decrease in 2013. The increment of small bowel cancer incidence correlated to other reports worldwide (Haselkorn et al., 2005; Bilimoria et al., 2009; Cahill et al., 2014; Anderson et al., 2015). In the present study, GIST was the most common histological subtype of small bowel cancer, followed by adenocarcinoma and lymphoma. This finding was not consistent with reports from the United States and China, which indicate that carcinoid and adenocarcinoma, respectively, are the most common histological subtypes. The incidence of small bowel cancer was more common in the United States than Asia. Unfortunately, the authors could not demonstrate the Thai incidence of small bowel malignancy in this study. However, there was evidence that the incidence of small bowel cancer increases with age, with it being most common between the ages of 61 and 70 years. This finding was similar to a U.S. report that asserted the mean age of diagnosis for any small bowel cancer to be 65 years, although sarcoma and lymphoma diagnoses tend to occur earlier. The incidence rises after the age of 40 years for all histological subtypes (Haselkorn et al., 2005).

Predisposing factors for small bowel cancer such as Crohn disease, excessive alcohol intake, smoking, or preexisting colorectal cancer were not found to be related in this study (data not shown). No patient in our study was previously diagnosed with Crohn disease, celiac disease, Peutz-Jeghers syndrome, familial adenomatous polyposis syndrome, or hereditary non-polyposis colorectal cancer.

The clinical manifestations of small bowel cancer are non-specific and disease onset varies, making diagnosis challenging. Patients diagnosed with adenocarcinoma or lymphoma develop symptoms earlier (2-3 mo after onset) than patients diagnosed with GIST (6 mos after onset). Further complicating diagnosis is the fact that a small subset of patients $(\sim 5 \%)$ are completely asymptomatic and about one-third of patients have normal physical examinations. Although weight loss, abdominal pain, and upper gastrointestinal bleeding are common symptoms of small bowel cancer, obstruction is a common symptom of adenocarcinoma. Moreover, anemia and upper gastrointestinal bleeding are symptoms specific to GIST and lymphoma. Interestingly, this study identified the most common site for GIST, adenocarcinoma, and lymphoma to be the jejunum, duodenum, and ileum, respectively.

There is no best technique to apply to each individual case of small bowel cancer. The authors demonstrated that ultrasonography and CT detect the disease at a sensitivity level of $67.4 \%$ and $76.2 \%$, respectively. Barium study (small bowel follow-through) has a sensitivity of 56\% to $87.4 \%$ to detect abnormal findings in the small bowel, including obstruction, ulcer, intestinal lumen narrowing, or filling defects (Ekberg and Ekholm, 1980; Martin et al., 1980; Paivansalo et al., 1988). Recently, video capsule endoscopy (VCE) was considered an alternative non-invasive investigation for diagnosis of small bowel tumor that might be missed by other strategies (Bailey, 2006). It had better sensitivity compared to barium study, especially for small bowel polyp detection (Mata et al., 2005). EGD is capable of detecting small bowel cancer localized to the duodenum and proximal jejunum, but its sensitivity is only $72.7 \%$.

In conclusion, small bowel cancers present late with non-specific symptoms. GIST is the most common histological subtype identified in Thailand, followed by adenocarcinoma and lymphoma. In this article, we have provided data detailing common locations, symptoms, tumor types, and tumor sizes associated with several common subtypes of small bowel cancer. In conjunction with the results from this study, radiologic and endoscopic techniques including EGD, ultrasound, and CT will help physicians diagnose cases of small bowel cancer earlier and with more accuracy, leading to improved patient outcomes.

\section{References}

Alkabie S, Bello B, Martinez RF, et al (2015). Metastatic adenocarcinoma of unknown origin presenting as small bowel perforation: a case report and literature review. $J$ Investig Med High Impact Case Rep, 3, 2324709615577415.

Anderson LA, Tavilla A, Brenner H, et al (2015). Survival for oesophageal, stomach and small intestine cancers in Europe 1999-2007: results from EUROCARE-5. Eur J Cancer, 5, 959-8049(15)00706-6.

Bailey AA, Debinski HS, Appleyard MN, et al (2006). Diagnosis and outcome of small bowel tumors found by capsule endoscopy: a three-center Australian experience. Am J Gastroenterol, 101, 2237-43.

Bennett CM, Coleman HG, Veal PG, et al (2015). Lifestyle factors and small intestine adenocarcinoma risk: A systematic review and meta-analysis. Cancer Epidemiol, 39, 265-73.

Bilimoria KY, Bentrem DJ, Wayne JD, et al (2009). Small bowel cancer in the United States: changes in epidemiology, treatment, and survival over the last 20 years. Ann Surg, 249, 63-71.

Cahill C, Gordon PH, Petrucci A, et al (2014). Small bowel adenocarcinoma and Crohn's disease: any further ahead than 50 years ago? World J Gastroenterol, 20, 11486-95.

Canavan C, Abrams KR, Hawthorne B, et al (2006). Long-term 
prognosis in Crohn's disease: factors that affect quality of life. Aliment Pharmacol Ther, 23, 377-85.

Conversano A, Macina S, Indellicato R, et al (2014). Gastrointestinal bleeding as presentation of small bowel metastases of malignant melanoma: Is surgery a good choice? Int J Surg Case Rep, 5, 774-8.

Ekberg O, Ekholm S (1980). Radiology in primary small bowel adenocarcinoma. Gastrointest Radiol, 5, 49-53.

Gorski RL, Jalil SA, Razick M, et al (2015). An obscure cause of gastrointestinal bleeding: Renal cell carcinoma metastasis to the small bowel. Int J Surg Case Rep, 15, 130-2.

Haselkorn T, Whittemore AS, Lilienfeld DE (2005). Incidence of small bowel cancer in the United States and worldwide: geographic, temporal, and racial differences. Cancer Causes Control, 16, 781-7.

Islam RS, Leighton JA, Pasha SF (2014). Evaluation and management of small-bowel tumors in the era of deep enteroscopy. Gastrointest Endosc, 79, 732-40.

Ismail I, Neuen BL, Mantha M (2015). Solitary jejunal metastasis from renal cell carcinoma presenting as small bowel obstruction 19 years after nephrectomy. BMJ Case Rep, 14, 2015.

Kouladouros K, Gartner D, Münch S, et al (2015). Recurrent intussusception as initial manifestation of primary intestinal melanoma: Case report and literature review. World $J$ Gastroenterol, 21, 3114-20.

Liu W, Zhou W, Qi WL, et al (2015). Gastrointestinal hemorrhage due to ileal metastasis from primary lung cancer. World $J$ Gastroenterol, 21, 3435-40.

Martin LF, Max MH, Richardson JD. (1980). Small bowel tumors: a continuing challenge. South Med J, 73, 981-5.

Mata A, Llach J, Castells A, et al (2005). A prospective trial comparing wireless capsule endoscopy and barium contrast series for small-bowel surveillance in hereditary GI polyposis syndromes. Gastrointest Endosc, 61, 721-5.

Paivansalo M, Siniluoto T, Jalovaara P (1988). Radiological findings in small-bowel tumours. Rofo, 149, 615-8.

Pan SY, Morrison H (2011). Epidemiology of cancer of the small intestine. World J Gastrointest Oncol, 3, 33-42.

Parkin DM, Muir CS, Whelan SL, et al (1992). Cancer incidence in five continents, volume VI. IARC Scientific Publications, Geneva p. 1033

Paski SC, Semrad CE (2009). Small bowel tumors. Gastrointest Endosc Clin N Am, 19, 461-79.

Qiu H, Yuan L, Ou Y, et al (2015). Small intestine metastasis from cervical cancer with acute abdomen: A case report. Oncol Lett, 9, 187-90.

Reynolds I, Healy P, Mcnamara DA (2014). Malignant tumours of the small intestine. Surgeon, 12, 263-70.

Zhou ZW, Wan DS, Chen G, et al (1999). Primary malignant tumor of the small intestine. World J Gastroenterol, 5, 273-6. 